\title{
Expression of epithelial-mesenchymal transition markers at the invasive front of oral squamous cell carcinoma
}

Liana Cristina Melo Carneiro COSTA ${ }^{1}$, Camila Ferreira LEITE¹, Sérgio Vitorino CARDOSO², Adriano Mota LOYOLA², Paulo Rogério de FARIA ${ }^{3}$, Paulo Eduardo Alencar SOUZA ${ }^{1}$, Martinho Campolina Rebello HORTA ${ }^{1}$

\footnotetext{
1- Department of Dentistry, Pontifícia Universidade Católica de Minas Gerais (PUC Minas), Belo Horizonte, MG, Brazil.

2- Laboratory of Oral and Maxillofacial Pathology, School of Dentistry, Federal University of Uberlândia (UFU), Uberlândia, MG, Brazil.

3- Institute of Biomedical Sciences, Federal University of Uberlândia (UFU), Uberlândia, MG, Brazil.
}

Corresponding address: Martinho Campolina Rebello Horta - Departamento de Odontologia - Pontifícia Universidade Católica de Minas Gerais - Av. Dom José Gaspar 500 - Prédio 45 - Sala 110 - Belo Horizonte - MG - Brazil - 30535-901 - Phone: (+55) 3133194341 - Fax: (+55) 31 33194166 - E-mail: martinhohorta@pucminas.br

Submitted: May 12, 2014 - Modification: October 27, 2014 - Accepted: January 28, 2015

\section{ABSTRACT}

\begin{abstract}
$\mathrm{O}$ ral squamous cell carcinoma (OSCC) is one of the most common malignances. In epithelial-mesenchymal transition (EMT), epithelial cells switch to mesenchymallike cells exhibiting high mobility. This migratory phenotype is significant during tumor invasion and metastasis. Objective: The aim of this study is to evaluate the expression of the EMT markers E-cadherin, N-cadherin and vimentin in OSCC. Material and Methods: Immunohistochemical detection of E-cadherin, $\mathrm{N}$-cadherin and vimentin was performed on 20 OSCC samples. Differences in the expression of each protein at the invasive front (IF) and in the central/superficial areas (CSA) of the tumor were assessed. Differences in the expression of each protein at the IF of both histologically high- and low-invasive OSCCs were evaluated. Associations among expression of proteins at the IF were assessed. Correlations between the expression levels of each protein at the IF and the tumor stage and clinical nodal status were also evaluated. Results: Reduced expression of E-cadherin was detected in 15 samples (75\%). E-cadherin expression was reduced at the IF when compared to the CSA and in high-invasive tumors when compared to low-invasive tumors. All samples were negative for $\mathrm{N}$-cadherin, even though one sample showed an inconspicuous expression. Positive expression of vimentin was observed in 6 samples (30\%). Nevertheless, there was no difference in vimentin expression between the IF and the CSA regions or between the low- and high-invasive tumors. Furthermore, no association was observed among protein expression levels at the IF. Finally, no correlations were observed between each protein's expression levels and tumor stage or clinical nodal status. Conclusions: Reduced E-cadherin expression at the IF and its association with histological invasiveness suggest that this protein is a noteworthy EMT marker in OSCC. Although vimentin was also detected as an EMT marker, its expression was neither limited to the IF nor was it related to histological invasiveness.
\end{abstract}

Keywords: Oral cancer. Squamous cell carcinoma. Cadherins. Vimentin.

\section{INTRODUCTION}

Oral squamous cell carcinoma (OSCC) is one of the most common malignances worldwide ${ }^{6}$, and its most significant risk factors are the use of tobacco and alcohol ${ }^{22}$. This tumor mostly affects men over 40 years old and frequently occurs in the lower lip, tongue, floor of the mouth, soft palate, and the gingival/alveolar ridge ${ }^{12}$. The deepest and most invasive areas of this tumor are histologically located at the invasive front (IF), which has been identified as the area chiefly responsible for its clinical behavior ${ }^{3,17}$.

The epithelial-mesenchymal transition (EMT) is a biological event in which epithelial cells lose many of their phenotypic features and gain extra properties 
typical of mesenchymal cells. One of the hallmark signs of EMT is the transformation of cohesive and polarized epithelial cells into mesenchymal-like cells that exhibit no polarization and high mobility. This migratory phenotype is the hallmark of an important type of EMT that involves neoplastic cells originating from epithelial malignant neoplasms during tumor invasion and metastasis $4,7,21,25,29$.

The transmembrane glycoprotein E-cadherin is the major molecule responsible for cell-cell adhesion in epithelial tissues and plays an essential role in the establishment and maintenance of its polarity and structural integrity ${ }^{2,24}$. A loss of E-cadherin expression increases the mobility of epithelial cells and its ability for local invasion, and has been used as a marker of EMT during tumor progression $^{2,7,21,24,29}$.

$\mathrm{N}$-cadherin is a member of the cadherin family and is located mainly in neural tissue and striated muscle ${ }^{2,24}$. Although epithelial cells do not express this molecule, a gain in $\mathrm{N}$-cadherin expression is usually associated with loss of E-cadherin expression during an EMT phenomenon called the "cadherin switch", which increases the mobility of neoplastic epithelial cells and their ability to invade locally .

Vimentin is a main component of the intermediate filament family of proteins, and it is expressed in mesenchymal cells such as fibroblasts, endothelial cells, hematopoietic cells and glial cells ${ }^{20}$. This protein is not expressed in normal epithelial cells and has been identified as a marker of EMT in epithelial malignant tumors. It is also associated with an invasive tumor cell phenotype 20,29.

The aim of this study is to evaluate the expression of the EMT markers E-cadherin, $\mathrm{N}$-cadherin and vimentin in OSCC. Differences in the expression of each protein at the IF and in central/ superficial areas (CSA) of the tumor were assessed. Differences in the expression of each protein at the IF of both high- and low-invasive OSCCs were also evaluated. Moreover, associations among protein expression levels at the IF were evaluated. Finally, correlations between the expression levels of each protein at the IF and the tumor stage and the clinical nodal status were also assessed.

\section{MATERIAL AND METHODS}

\section{Tissue samples}

This study was approved by the local ethics committee (CAAE - 0174.0.213.000-10).

A total of 20 OSCC samples were obtained from archival formalin-fixed paraffin-embedded specimens and were evaluated. Out of the 20 OSCC samples, 18 (90\%) were from male and 2 (10\%) from female individuals. The age of the patients ranged from 34 to 73 years (mean age: 54.2 years).
Out of the 20 tumors, 7 were sited at the tongue, 4 at the lower lip, 4 at the floor of the mouth, 2 at the retromolar triangle, 2 at the soft palate and 1 at the alveolar ridge. Tumors were staged by the TNM classification, as follows: 5 stage I tumors, 5 stage II tumors, 4 stage III tumors and 6 stage IV tumors. Based on the $\mathrm{N}$ stage of the TNM, the clinical nodal status of the tumors was also categorized as follows: absence of clinical nodal metastasis (NO; 13 tumors) or presence of clinical nodal metastasis (N1, N2 or N3; 7 tumors).

The following inclusion criteria were applied: 1) OSCC samples were obtained by total surgical resection; and 2) samples were limited to OSCCs in which the CSA and the IF could be clearly identified.

\section{Evaluation of OSCC patterns of invasion}

The patterns of invasion of OSSC samples were examined in hematoxylin and eosin stained sections according to the method of Bryne, et al. ${ }^{3}$ (1992). Each pattern of invasion was classified as one of the following: grade 1 - pushing, well delineated infiltrating borders; grade 2 - infiltrating, solid cords, bands and/or strands; grade 3 - small groups or cords of infiltrating cells ( $n>15$ cells); and grade 4 - marked and widespread cellular dissociation in small groups and/or in single cells ( $n<15$ cells). The samples were subsequently classified as either low-invasive OSCC (pattern of invasion grades 1 , 2 or 3 ) or high-invasive OSCC (pattern of invasion grade 4 ), as previously reported ${ }^{23}$.

\section{Immunohistochemistry}

For the immunohistochemical detection of $\mathrm{E}$-cadherin, $\mathrm{N}$-cadherin and vimentin, $4 \mu \mathrm{m}$ sections obtained from the paraffin-embedded samples were used. Tissue sections were dewaxed with xylenes, hydrated with graded alcohols, and treated with $0.6 \% \mathrm{H}_{2} \mathrm{O}_{2}$ to eliminate endogenous peroxidase activity. Antigen retrieval for E-cadherin and $\mathrm{N}$-cadherin was performed by heating the samples in a $0.01 \mathrm{M}$ citrate buffer ( $\mathrm{pH} 6.0$ ) for 30 minutes. Antigen retrieval for vimentin was performed by heating the samples in a Tris/EDTA buffer $(\mathrm{pH} 9.0)$ for 30 minutes. Subsequently, one of the following primary antibodies was used: 1) anti-E-cadherin monoclonal antibody (clone 36B5, diluted 1:50, incubated overnight at $4^{\circ} \mathrm{C}$; Leica Biosystems, Newcastle, UK); 2) anti-N-cadherin monoclonal antibody (clone IAR06, diluted 1:50, incubated overnight at $4^{\circ} \mathrm{C}$; Leica Biosystems, Newcastle, UK); or 3) anti-vimentin monoclonal antibody (clone SRL33, diluted 1:200, incubated overnight at $4^{\circ} \mathrm{C}$; Leica Biosystems, Newcastle, UK). Afterward, one of the following immunohistochemical visualization systems was used (according to the manufacturer's instructions): 1) For E-cadherin and vimentin detection, the LSAB+ kit (Dako Corporation, 
Carpinteria, California, USA); 2) For N-cadherin detection, the REVEAL Biotin-Free Detection System (Spring Bioscience Corporation, Pleasanton, California, USA). The reactive products were visualized by immersing the sections in $0.03 \%$ diaminobenzidine solution containing $2 \mathrm{mM} \mathrm{H}_{2} \mathrm{O}_{2}$ for 3 min The sections were then counterstained with Mayer's hematoxylin, dehydrated, and mounted.

Samples of oral fibrous hyperplasia were used as positive controls for E-cadherin and vimentin staining. In addition, samples of normal cardiac striated muscle and normal testis were used as positive controls for $\mathrm{N}$-cadherin. Furthermore, immunostaining of morphologically normal oral mucosa epithelium adjacent to the tumor, peripheral nerve bundles in tumor stroma and the tumor's connective tissue mesenchymal cells were used as internal positive controls for $\mathrm{E}$-cadherin, $\mathrm{N}$-cadherin and vimentin, respectively. Negative controls were performed via omission of the primary antibody.

\section{Scoring of immunostaining results}

The expression of $\mathrm{E}$-cadherin, $\mathrm{N}$-cadherin and vimentin in each sample was independently assessed at the IF and at the CSA.

The expression of E-cadherin was classified as follows ${ }^{14}$ : 1) "preserved": membranous immunostaining in more than $50 \%$ of the epithelial tumor cells; or 2) "reduced": membranous immunostaining in $50 \%$ or less of the epithelial tumor cells.

The expression of $\mathrm{N}$-cadherin was classified as follows 19: 1) "negative": cytoplasmic or membranous immunostaining in less than $5 \%$ of the epithelial tumor cells; or 2) "positive": cytoplasmic or membranous immunostaining in $5 \%$ or more of the epithelial tumor cells.

The expression of vimentin was classified as follows ${ }^{27}: 1$ ) "negative": cytoplasmic immunostaining in less than $10 \%$ of the epithelial tumor cells; or 2 ) "positive": cytoplasmic immunostaining in $10 \%$ or more of the epithelial tumor cells.

This analysis was performed independently by two authors. Doubtful cases were reanalyzed, and a consensus score was agreed upon.

\section{Statistical analysis}

Differences in the expression levels of each protein at the IF and the CSA of the OSCC were analyzed using the McNemar test. Fischer's exact test was used to assess differences in the expression levels of each protein at the IF for both high- and low-invasive OSCC. Fischer's exact test was used to evaluate associations between the expression levels of each protein at the IF. The Spearman test was employed to assess correlations between the expression levels of each protein at the IF and the tumor stage (TNM classification). Finally, Fischer's

Table 1- E-cadherin, $\mathrm{N}$-cadherin and vimentin expression at the invasive front (IF) and in the central/superficial areas (CSA) of oral squamous cell carcinoma

\begin{tabular}{|c|c|c|}
\hline E-cadherin expression & $\mathbf{n}$ & p-value* \\
\hline Preserved at the CSA / Preserved at the IF & $5(25 \%)$ & $<0.05$ \\
\hline Preserved at the CSA / Reduced at the IF & $11(55 \%)$ & \\
\hline Reduced at the CSA / Preserved at the IF & $0(0 \%)$ & \\
\hline Reduced at the CSA / Reduced at the IF & $4(20 \%)$ & \\
\hline Total & $20(100 \%)$ & \\
\hline $\mathrm{N}$-cadherin expression & $\mathrm{n}$ & $p$-value* \\
\hline Negative at the CSA / Negative at the IF & $20(100 \%)$ & n.s. \\
\hline Negative at the CSA / Positive at the IF & $0(0 \%)$ & \\
\hline Positive at the CSA / Negative at the IF & $0(0 \%)$ & \\
\hline Positive at the CSA / Positive at the IF & $0(0 \%)$ & \\
\hline Total & $20(100 \%)$ & \\
\hline Vimentin expression & $\mathrm{n}$ & p-value* \\
\hline Negative at the CSA / Negative at the IF & $14(70 \%)$ & n.s. \\
\hline Negative at the CSA / Positive at the IF & $3(15 \%)$ & \\
\hline Positive at the CSA / Negative at the IF & $0(0 \%)$ & \\
\hline Positive at the CSA / Positive at the IF & $3(15 \%)$ & \\
\hline Total & $20(100 \%)$ & \\
\hline
\end{tabular}

*p-values were obtained by the McNemar test

n.s. - not significant 
exact test was also used to evaluate associations between the expression levels of each protein at the IF and the clinical nodal status. The level of significance was set at $5 \%$. All statistical analysis was performed using GraphPad Prism software (GraphPad Software, San Diego, California, USA).

\section{RESULTS}

Regarding the pattern of invasion, the 20 OSCC samples were initially classified as grade 1 (1 sample), grade 2 (1 sample), grade 3 (5 samples) and grade 4 (13 samples). Afterward, these scores were dichotomized as 7 low-invasive OSCCs (pattern of invasion grades 1,2 or 3) and 13 high-invasive OSCCs (pattern of invasion grade 4 ).

The results of the E-cadherin expression are illustrated in both Table 1 and Figure 1 . Out of the 20 OSCC samples, 15 (75\%) exhibited reduced expression of $\mathrm{E}$-cadherin in one of the two evaluated regions (CSA or IF). Preserved E-cadherin expression at both the CSA and the IF was observed in 5 (25\%) samples. Preserved E-cadherin expression at the CSA and reduced expression at the IF were found in 11 (55\%) samples. Reduced E-cadherin expression at both the CSA and the IF of OSCCs was seen in $4(20 \%)$ samples. Samples exhibiting reduced $\mathrm{E}$-cadherin expression at the
CSA and preserved expression at the IF were not observed. The E-cadherin expression was reduced at the IF when compared to expression levels at the CSA $(p<0.05)$.

The $\mathrm{N}$-cadherin expression is illustrated in both Table 1 and Figure 2. N-cadherin expression was negative in all 20 of the OSCC samples at both the IF and the CSA, and no statistically significant difference was observed ( $p>0.05$ ). It is important to inform that one OSCC sample showed an inconspicuous $\mathrm{N}$-cadherin expression. These few tumor cells expressing $\mathrm{N}$-cadherin were located in a minor area at the IF of a high-invasive tumor (pattern of invasion grade 4). Nevertheless, even this sample was classified as negative for $\mathrm{N}$-cadherin, since less than $5 \%$ of the epithelial tumor cells in this area showed immunostaining.

Vimentin expression results are demonstrated in both Table 1 and Figure 3. Out of the 20 OSCC samples, $6(30 \%)$ exhibited positive vimentin expression in one of the two evaluated regions (CSA or IF). Negative vimentin expression at both the CSA and the IF was observed in 14 (70\%) samples. Negative vimentin expression at the CSA and positive expression at the IF was seen in 3 (15\%) samples. Positive vimentin expression at both the CSA and the IF was found in $3(15 \%)$ samples. No samples exhibited positive vimentin expression
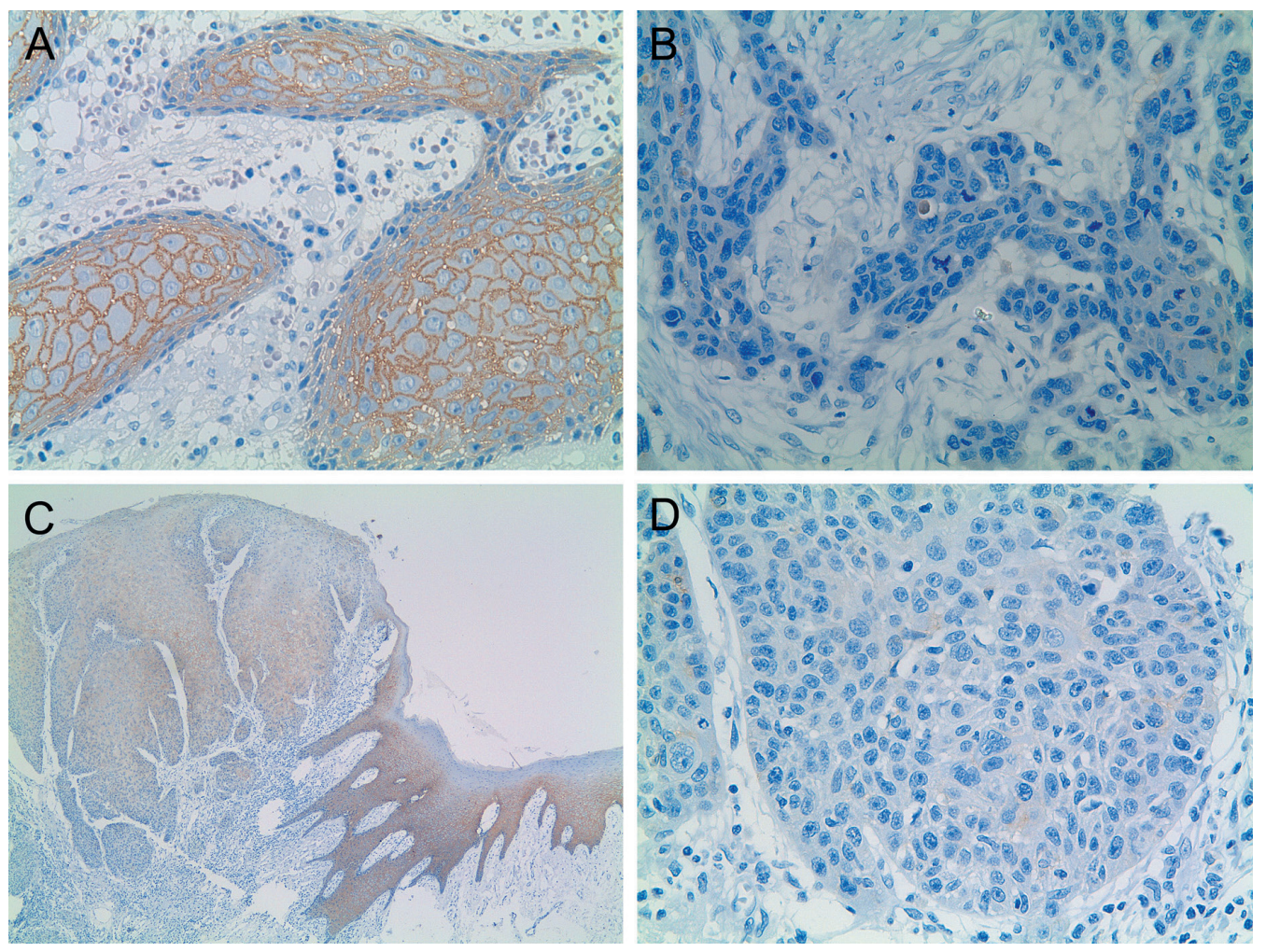

Figure 1- Immunohistochemical reactivity for E-cadherin in oral squamous cell carcinoma (OSCC). A: OSCC displaying preserved E-cadherin expression at the CSA (X400); B: OSCC exhibiting reduced E-cadherin expression at the IF (X400); $\mathrm{C}$ and D: OSCC exhibiting preserved E-cadherin expression at the morphologically normal oral mucosa epithelium adjacent to the tumor $(\mathrm{C}, \mathrm{X} 40)$, preserved E-cadherin expression at the CSA (C, X40) and reduced E-cadherin expression at the IF (C, X40; D, X400) 

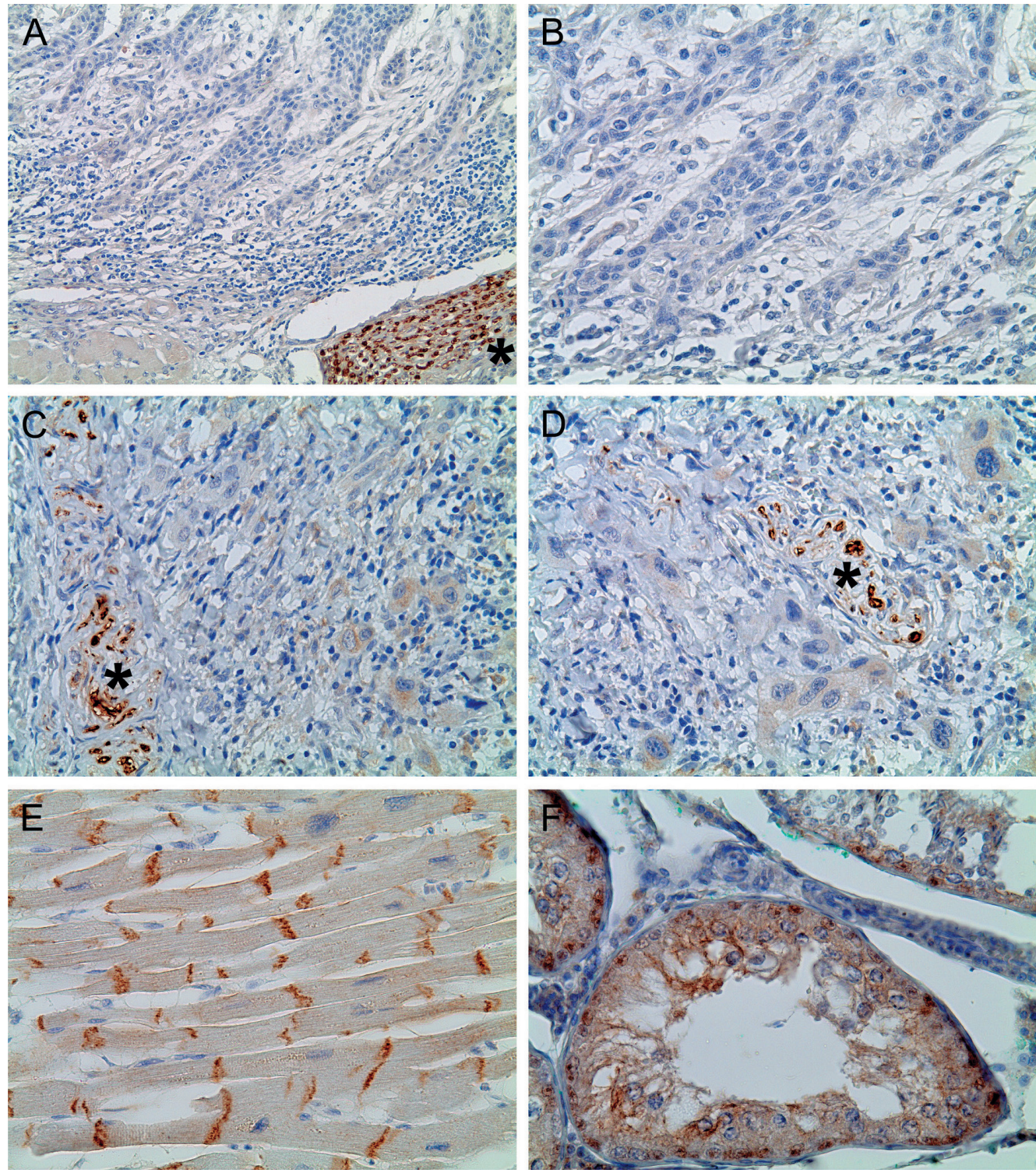

Figure 2- Immunohistochemical reactivity for $\mathrm{N}$-cadherin in oral squamous cell carcinoma (OSCC), normal cardiac striated muscle and normal testis. In OSCC, immunostaining of peripheral nerve bundles in the tumor stroma (marked with asterisk) were used as internal positive controls for N-cadherin. A and B: OSCC exhibiting negative N-cadherin expression (A, X200; $B, X 400) ; C$ and D: OSCC showing inconspicuous N-cadherin expression (C, X400; D, X400); E: normal cardiac striated muscle ( $\mathrm{N}$-cadherin positive control) exhibiting positive $\mathrm{N}$-cadherin immunostaining in the intercalated disc (E, X400); $\mathrm{F}$ : normal testis ( $\mathrm{N}$-cadherin positive control) showing cytoplasmic and membranous $\mathrm{N}$-cadherin immunostaining (E, X400)

at the CSA and negative expression at the IF. No statistically significant difference was observed for vimentin expression when the IF region was compared to the CSA region $(p>0.05)$.

The comparison of each protein expression level at the IF for both high- and low-invasive OSCC is illustrated in Table 2. The E-cadherin expression was reduced at the IF of high-invasive OSCC when compared to low-invasive OSCCs $(p<0.05)$. Nevertheless, no difference in either vimentin expression at the IF or $\mathrm{N}$-cadherin expression at the IF was observed for low- and high-invasive OSCCs ( $p>0.05)$.

Results of the association between the expression levels of each protein at the IF are illustrated in Table 3. As shown, no association was observed $(p>0.05)$.

Finally, no correlations were observed between the expression levels of each protein at the IF and the tumor stage (TNM classification) $(p>0.05)$ or the clinical nodal status (based on the $\mathrm{N}$ stage of the TNM) ( $p>0.05)$.

\section{DISCUSSION}

EMT is an important biological event in which cohesive and polarized epithelial cells switch to mesenchymal-like cells exhibiting no polarization 

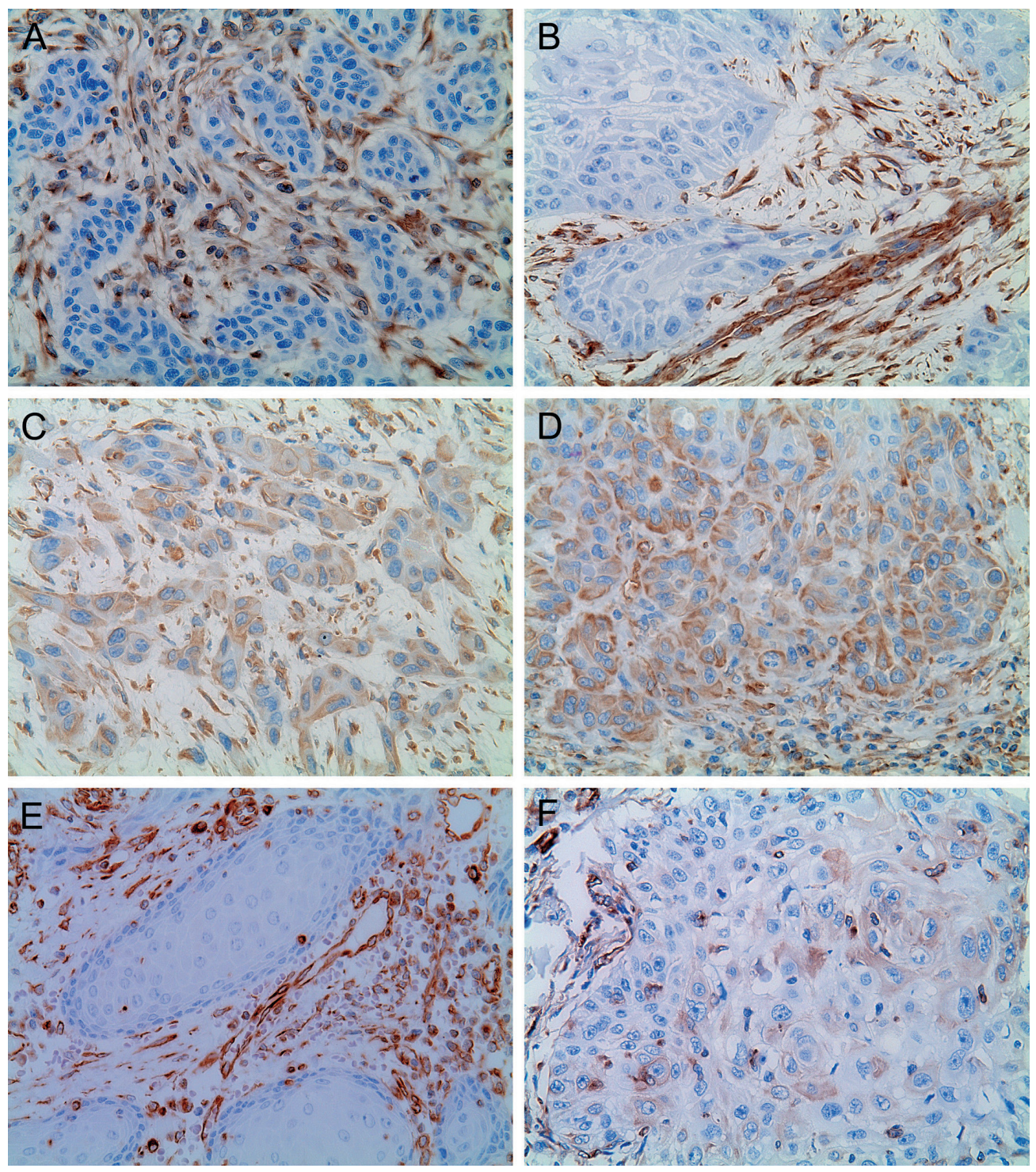

Figure 3- Immunohistochemical reactivity for vimentin in oral squamous cell carcinoma (OSCC). Immunostaining of the tumor's connective tissue mesenchymal cells was used as an internal positive control. A and B: OSCC showing negative vimentin expression (A, X400; B, X400); C and D: OSCC displaying positive vimentin expression at the IF (E, X400; F, $X 400)$; $E$ : OSCC exhibiting negative vimentin expression at the CSA (E, X400). This is the same sample of the Figure $1 \mathrm{~A}$ (preserved E-cadherin expression); F: OSCC displaying positive vimentin expression at the IF (E, X400). This is the same sample of the Figure 1D (reduced E-cadherin expression)

and high mobility. This acquired migratory phenotype is important, particularly during tumor invasion and metastasis $4,7,21,25,29$. The altered expression of EMT markers such as E-cadherin, $\mathrm{N}$-cadherin and vimentin have recently been studied in various malignant epithelial tumors ${ }^{7,29}$. Because the IF has been identified as the region chiefly responsible for the clinical behavior of OSCC 3,17 , the evaluation of these EMT markers in this tumor area is noteworthy.

This study revealed a heterogeneous loss of E-cadherin expression in OSCCS, as reduced expression of this protein was observed in 15 of the samples $(75 \%)$. Reduced expression of E-cadherin has been previously reported in
OSCCS ${ }^{1,5,8,13,14,16,19,26-28}$. E-cadherin is the main adhesion molecule in epithelial tissue and is essential for the establishment and maintenance of epithelial structural integrity ${ }^{2,24}$. Several mechanisms for inactivating E-cadherin protein or the $\mathrm{E}$-cadherin gene $\mathrm{CDH} 1$ have been described in human malignant tumors: hereditary and somatic mutations, promoter hypermethylation, abnormal protein processing, and induction of transcriptional repressors ${ }^{2}$. Because the loss of $\mathrm{E}$-cadherin expression increases the mobility of epithelial cells and its ability to infiltrate locally, it has been used as an EMT marker during tumor invasion and metastasis $2,7,24,29$. Loss of $\mathrm{E}$-cadherin expression in OSCCs has been associated with a reduction 
Table 2- E-cadherin, N-cadherin and vimentin expression at the invasive front (IF) of low- and high-invasive oral squamous cell carcinoma

\begin{tabular}{lccc}
\hline & Low-invasive OSCC & High-invasive OSCC & p-value * \\
\hline E-cadherin & $4(20 \%)$ & & $<0.05$ \\
Preserved & $3(15 \%)$ & $1(5 \%)$ & n.s. \\
Reduced & & & \\
N-cadherin & $7(35 \%)$ & $13(65 \%)$ & \\
Negative & $0(0 \%)$ & $0(0 \%)$ & n.s. \\
Positive & & & \\
Vimentin & $6(30 \%)$ & $5(40 \%)$ & \\
Negative & $1(5 \%)$ & & \\
\hline Positive & & & \\
\hline
\end{tabular}

* $p$-values were obtained by Fischer's Exact test

n.s. - not significant

OSCC=oral squamous cell carcinoma

Table 3- E-cadherin, N-cadherin and vimentin expression at the invasive front (IF) of oral squamous cell carcinoma

\begin{tabular}{lccc}
\hline & Vimentin (negative) & Vimentin (positive) & p-value* $^{*}$ \\
\hline E-cadherin (preserved) & $5(25 \%)$ & $0(0 \%)$ & n.s. \\
E-cadherin (reduced) & $9(45 \%)$ & $6(30 \%)$ & p-value* \\
N-cadherin (negative) & Vimentin (negative) & Vimentin (positive) & n.s. \\
N-cadherin (positive) & $14(70 \%)$ & $6(30 \%)$ & p-value* \\
E-cadherin (preserved) & $0(0 \%)$ & $0(0 \%)$ & n.s. \\
E-cadherin (reduced) & N-cadherin (negative) & N-cadherin (positive) & $0(0 \%)$ \\
\hline
\end{tabular}

* p-values were obtained by Fischer's Exact test

n.s. - not significant

in disease-free rates ${ }^{1,5}$, a higher prevalence of lymph node metastasis ${ }^{19,27}$ and a lower survival rate $1,5,13,27,28$, although no association with prognosis has been reported ${ }^{14,16,26}$.

Reduced E-cadherin expression was observed at the IF when compared to the CSA of the tumor $(p<0.05)$. Similar results have been previously reported ${ }^{19,27,28}$. These findings advocate that the loss of E-cadherin expression occurs mainly at the IF, suggesting that tumor cells in this important area could detach and invade local tissue more easily and thus increase the potential for metastasis.

E-cadherin expression was reduced at the IF of high-invasive OSCCs when compared to lowinvasive OSCCs $(p<0.05)$. This result suggests that its reduction at the IF may be associated with a high histological dissociation pattern of the tumor cells. It is important to note that the histological pattern of invasion, which is used to categorize the tumors as low- or high-invasive, is an important histological parameter that reflects the invasive features of the tumor ${ }^{3,17}$ and has been used as a prognostic marker for OSCC ${ }^{17}$. Diniz-Freitas, et al. ${ }^{5}$ (2006) demonstrated that weak or absent E-cadherin expression in OSCC was associated with a more invasive histological pattern. Moreover, Wang, et al. $^{28}$ (2009) reported that OSCC exhibiting loss of E-cadherin expression at the IF also showed the highest IF histological grading scores. In contrast, Mahomed, et al.14 (2007) did not observe an association between E-cadherin expression at the IF and the IF grading score in OSCC samples.

All OSCC samples were negative for $\mathrm{N}$-cadherin, despite the fact that one sample showed an inconspicuous expression. Although some studies have described very limited expression of $\mathrm{N}$-cadherin in OSCC, it was not correlated with clinicopathological parameters ${ }^{8,26}$; however, an association between $\mathrm{N}$-cadherin expression in OSCC and lymph node metastasis was reported ${ }^{30}$. These divergent results for $\mathrm{N}$-cadherin expression obtained during the present study and previous 
reports that have evaluated OSCC could be explained by discrepancies in sample processing, the use of different primary antibodies, variability in immunohistochemical techniques or even the immunostaining evaluation employed in each study. It is important to state that in the present study, $\mathrm{N}$-cadherin expression was rigorously assessed using appropriate external and internal positive controls in selected OSCC samples that were subjected to the criteria outlined in the Material and Methods section.

As an obvious consequence of the absence of $\mathrm{N}$-cadherin expression, the present study demonstrates no association between E-cadherin and $\mathrm{N}$-cadherin expression at the IF. Therefore, no occurrence of an $\mathrm{E}$-cadherin to $\mathrm{N}$-cadherin switch was observed in the OSCC samples evaluated in this study. The concomitant loss of E-cadherin and gain of $\mathrm{N}$-cadherin expression (cadherin switch) has been recently reported in malignant epithelial tumors including head and neck $^{30}$ and oral ${ }^{19}$ squamous cell carcinoma. This phenomenon, which increases both the mobility and invasiveness of neoplastic epithelial cells, has also been used as an indicator of the occurrence of EMT ${ }^{19}$. Nevertheless, unrelated loss of $\mathrm{E}$-cadherin and gain of $\mathrm{N}$-cadherin, not giving rise to cadherin switch, has also been reported in $\mathrm{OSCC}^{8,26}$. In fact, in vitro studies have demonstrated that overexpression of Slug (a member of the Snail family of zinc finger transcription factors) induces an $\mathrm{E}$ - to $\mathrm{N}$-cadherin switch in oral ${ }^{11}$ and head and neck ${ }^{30}$ squamous cell carcinoma cell lines. However, as demonstrated by Hashimoto, et al. ${ }^{8}$ (2012), OSCC cell lines expressing $\mathrm{N}$-cadherin in vitro produce xenotransplantated mouse tongue tumors showing an insignificant expression of $\mathrm{N}$-cadherin in vivo. According to these authors, the environments surrounding OSCC cells largely affect the $\mathrm{N}$-cadherin expression ${ }^{8}$.

A heterogeneous vimentin expression was observed in the present study, since positive expression of this EMT marker was observed in 6 samples (30\%). Similar results have been previously reported in OSCC ${ }^{13,27}$. Vimentin is an intermediate filament normally expressed by cells presenting a mesenchymal phenotype ${ }^{20}$. Its expression by epithelial malignant tumors cells is associated with high invasiveness and has been considered a reliable EMT marker ${ }^{20,29}$. In vitro studies have shown that inhibition of vimentin expression results in reversal of the mesenchymal phenotype and reduction of migratory and invasive capability of OSCC cell lines ${ }^{18}$. In fact, vimentin expression has been associated with higher prevalence of lymph node metastasis in esophageal ${ }^{10}$ as well as in head and neck squamous cell carcinoma ${ }^{15}$. Moreover, Liu, et al. ${ }^{13}$ (2010) reported a higher prevalence of recurrences and a lesser survival time in OSCC showing positive vimentin expression.

No significant difference in vimentin expression was observed between the IF and the CSA regions $(p>0.05)$; however, negative vimentin expression at the CSA and positive expression at the IF were observed in $3(15 \%)$ samples, and no samples exhibited positive vimentin expression at the CSA and negative expression at the IF. This finding suggests that the expression of this EMT marker in OSCCs is not limited to the IF cells. In contrast, Wang, et al. ${ }^{27}$ (2011) reported increased expression of vimentin at the IF of OSCCs when compared to the CSA of the tumor.

No difference in vimentin expression at the IF was observed between low- and high-invasive OSCC ( $p>0.05)$. Liu, et al. ${ }^{13}$ (2010) also reported no association between vimentin expression at the IF and the histological pattern of invasion of OSCC. These findings suggest that vimentin expression is not related to the histological invasiveness of the OSCC. Nevertheless, association between higher histological invasiveness and positive vimentin expression was observed in head and neck squamous cell carcinoma ${ }^{15}$.

No association was observed between E-cadherin, $\mathrm{N}$-cadherin and vimentin expression at the IF $(p>0.05)$. This result suggests that although EMT markers should mediate tumor progression ${ }^{21}$, the expression of different EMT markers in OSCC is not necessarily associated. As previously discussed, the concomitant loss of E-cadherin and gain of $\mathrm{N}$-cadherin (cadherin switch) was found in head and neck ${ }^{30}$ and oral ${ }^{19}$ squamous cell carcinoma; however, unrelated loss of $\mathrm{E}$-cadherin and gain of $\mathrm{N}$-cadherin in OSCC was also reported ${ }^{8,26}$. For E-cadherin and vimentin, it is important to note that, although no significant difference was observed, all samples that exhibited positive expression of vimentin at the IF also displayed reduced E-cadherin expression. In fact, Liu, et al. ${ }^{13}$ (2010) reported a correlation between loss of E-cadherin expression and gain of vimentin expression at the IF in OSCC.

No correlations were observed between the expression levels of each protein at the IF and the tumor stage $(p>0.05)$ or the clinical nodal status $(p>0.05)$. As previously discussed, loss of E-cadherin, gain of vimentin and gain of $\mathrm{N}$-cadherin in OSCC have been associated with a higher occurrence of lymph node metastasis ${ }^{19,27,30}$

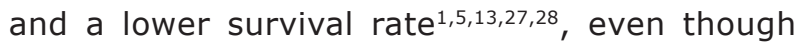
no association with prognosis has also been reported $8,14,16,26$. In fact, in the present study, the reduced sample size and the absence of survival data are limitations to these analyses.

Finally, it is important to explain the dichotomization of the pattern of invasion grades in low-invasive OSCCs (grades 1, 2 or 3 ) and high-invasive OSCCs (grade 4 ). This cut-off was 
used by Søland, et al.23 (2008), which found a worst prognostic outcome for patients with tumors showing a grade 4 pattern of invasion (marked and widespread cellular dissociation). Also regarding this classification, the fact that the number of high-invasive OCCCs (13 samples) was almost the double of low-invasive OSCCs (7 samples) should be considered a limitation of the study. Nevertheless, is noteworthy that the sample was composed of tumors removed by means of total surgical resection (and not by incisional biopsies). Therefore, since the whole tumor could be evaluated, the probability to identify areas showing marked and widespread cellular dissociation (grade 4 pattern of invasion) is supposed to be higher.

\section{CONCLUSION}

The reduced E-cadherin expression at the IF and its association with histological invasiveness suggest that this protein is a noteworthy EMT marker in OSCC. These findings reinforce the relevance of E-cadherin loss as a marker of EMT during OSCC progression because the IF has been identified as the region chiefly responsible for its behavior. Although vimentin was also detected as an EMT marker, its expression was neither limited to the IF nor related to histological invasiveness.

\section{ACKNOWLEDGEMENTS}

This study was supported by grants from National Council for Scientific and Technological Development (CNPq; 483717/2010-0), Fundação de Amparo à Pesquisa do Estado de Minas Gerais (FAPEMIG), and PUC Minas (FIP 2011/5859-1S), Brazil. The authors wish to thank the invaluable technical assistance provided by Mrs. Maria Reni Gonçalves Moitinha.

\section{REFERENCES}

1- Bánkfalvi A, Krassort M, Buchwalow IB, Végh A, Felszeghy E, Piffkó J. Gains and losses of adhesion molecules (CD44, E-cadherin, and $\beta$-catenin) during oral carcinogenesis and tumour progression. J Pathol. 2002;198:343-51.

2- Berx G, van Roy F. Involvement of members of the cadherin superfamily in cancer. Cold Spring Harb Perspect Biol. 2009;1:a003129.

3- Bryne M, Koppang HS, Lilleng R, Kjaerheim A. Malignancy grading of the deep invasive margins of oral squamous cell carcinomas has high prognostic value. J Pathol. 1992;166:375-81. 4- Chaffer CL, Thompson EW, Willians ED. Mesenchymal to epithelial transition in development and disease. Cells Tissues Organs. 2007;185:7-19.

5- Diniz-Freitas M, García-Caballero T, Antúnez-López J, GándaraRey JM, García-García A. Reduced E-cadherin expression is an indicator of unfavourable prognosis in oral squamous cell carcinoma. Oral Oncol. 2006;42:190-200.

6- Ferlay J, Shin HR, Bray F, Forman D, Mathers C, Parkin DM. Estimates of worldwide burden of cancer in 2008: GLOBOCAN 2008. Int J Cancer. 2010;127:2893-917.
7- Guarino M, Rubino B, Ballabio G. The role of epithelialmesenchymal transition in cancer pathology. Pathology. 2007;39:305-18.

8- Hashimoto T, Soeno Y, Maeda G, Taya Y, Aoba T, Nasu M, et al. Progression of oral squamous cell carcinoma accompanied with reduced E-cadherin expression but not cadherin switch. PLoS One. 2012;7:e47899.

9- Hazan RB, Qiao R, Keren R, Badano I, Suyama K. Cadherin switch in tumor progression. Ann N Y Acad Sci. 2004;1014:155-63. 10- Jin H, Morohashi S, Sato F, Kudo Y, Akasaka H, Tsutsumi S, et al. Vimentin expression of esophageal squamous cell carcinoma and its aggressive potential for lymph node metastasis. Biomed Res. 2010;31:105-12.

11- Katafiasz D, Smith LM, Wahl JK $3^{\text {rd }}$. Slug (SNAI2) expression in oral SCC cells results in altered cell-cell adhesion and increased motility. Cell Adh Migr. 2011;5:315-22.

12- Krolls SO, Hoffman S. Squamous cell carcinoma of the oral soft tissues: a statistical analysis of 14,235 cases by age, sex, and race of patients. J Am Dent Assoc. 1976;92:571-4.

13- Liu LK, Jiang $X Y$, Zhou $X X$, Wang $D M$, Song $X L$, Jiang $H B$. Upregulation of vimentin and aberrant expression of E-cadherin/ beta-catenin complex in oral squamous cell carcinomas: correlation with the clinicopathological features and patient outcome. Mod Pathol. 2010;23:213-24.

14- Mahomed F, Altini M, Meer S. Altered E-cadherin/ $\beta$-catenin expression in oral squamous carcinoma with and without nodal metastasis. Oral Dis. 2007;13:386-92

15- Mandal M, Myers JN, Lippman SM, Johnson FM, Williams MD, Rayala $S$, et al. Epithelial to mesenchymal transition in head and neck squamous carcinoma: association of Src activation with E-cadherin down-regulation, vimentin expression, and aggressive tumor features. Cancer. 2008;112:2088-100.

16- Mostaan LV, Khorsandi MT, Sharifian SM, Shandiz FH, Mirashrafi $F$, Sabzari $H$, et al. Correlation between E-cadherin and CD44 adhesion molecules expression and cervical lymph node metastasis in oral tongue SCC: predictive significance or not. Pathol Res Pract. 2011;207:448-51.

17- Odell EW, Jani P, Sherriff M, Ahluwalia SM, Hibbert J, Levison $\mathrm{DA}$, et al. The prognostic value of individual histologic grading parameters in small lingual squamous cell carcinomas. The importance of the pattern of invasion. Cancer. 1994;74:789-94. 18- Paccione RJ, Miyazaki H, Patel V, Waseem A, Gutkind JS, Zehner ZE, et al. Keratin down-regulation in vimentin-positive cancer cells is reversible by vimentin RNA interference, which inhibits growth and motility. Mol Cancer Ther. 2008;7:2894-903. 19- Pyo SW, Hashimoto M, Kim YS, Kim CH, Lee SH, Johnson KR, et al. Expression of E-cadherin, $\mathrm{P}$-cadherin and $\mathrm{N}$-cadherin in oral squamous cell carcinoma: correlation with the clinicopathologic features and patient outcome. J Craniomaxillofac Surg. 2007;35:19.

20- Satelli A, Li S. Vimentin in cancer and its potential as a molecular target for cancer therapy. Cell Mol Life Sci. 2011;68:3033-46

21- Scanlon CS, van Tubergen EA, Inglehart RC, D'Silva NJ. Biomarkers of epithelial-mesenchymal transition in squamous cell carcinoma. J Dent Res. 2013,92:114-21.

22- Scully C. Oral cancer aetiopathogenesis; past, present and future aspects. Med Oral Patol Oral Cir Bucal. 2011; 16:e306-11. 23- Søland TM, Brusevold IJ, Koppang HS, Schenck K, Bryne M. Nerve growth factor receptor (p75 NTR) and pattern of invasion predict poor prognosis in oral squamous cell carcinoma. Histopathology. 2008;53:62-72.

24- Stemmler MP. Cadherins in development and cancer. Mol Biosyst. 2008;4:835-50.

25- Thiery JP, Sleeman JP. Complex networks orchestrate epithelialmesenchymal transitions. Nat Rev Mol Cell Biol. 2006;7:131-42. 
26- Ukpo OC, Thorstad WL, Zhang Q, Lewis JS Jr. Lack of association of cadherin expression and histopathologic type, metastasis, or patient outcome in oropharyngeal squamous cell carcinoma: a tissue microarray study. Head Neck Pathol. 2012;6:38-47.

27- Wang $C$, Huang $H$, Huang $Z$, Wang A, Chen $X$, Huang $L$, et al. Tumor budding correlates with poor prognosis and epithelialmesenchymal transition in tongue squamous cell carcinoma. J Oral Pathol Med. 2011;40:545-55.
28- Wang X, Zhang J, Fan M, Zhou Q, Deng H, Aisharif MJ, et al. The expression of $\mathrm{E}$-cadherin at the invasive tumor front of oral squamous cell carcinoma: immunohistochemical and RT-PCR analysis with clinicopathological correlation. Oral Surg Oral Med Oral Pathol Oral Radiol Endod. 2009;107:547-54.

29- Zeisberg M, Neilson EG. Biomarkers for epithelial-mesenchymal transitions. J Clin Invest. 2009;119:1429-37.

30- Zhang J, Cheng Q, Zhou Y, Wang $Y$, Chen X. Slug is a key mediator of hypoxia induced cadherin switch in HNSCC: correlations with poor prognosis. Oral Oncol. 2013;49:1043-50. 